\title{
THE LOCATION OF THE ZEROS OF THE DERIVATIVES OF FABER POLYNOMIALS
}

\author{
J. L. ULLMAN
}

Abstract. We prove a property of Faber polynomials which supports a conjecture concerning the zeros of Faber polynomials.

1. Introduction. Let $K$ be a compact set consisting of more than one point having a connected and simply connected complement, $\tilde{K}$. Let $f(z)$ be the schlicht function mapping $\widetilde{K}$ onto the exterior of the circle $\Sigma_{w}=$ $\{w:|w|=1\}$, normalized so that

$$
f(z)=z+a_{0}+a_{-1} z^{-1}+\cdots .
$$

This normalization can always be achieved by changing units appropriately in the $z$ plane, and we assume that this is done.

The polynomial part of $(f(z))^{n}, F_{n}(z)$, is called the Faber polynomial of degree $n$ associated with $K$. There is a conjecture that for each $n, n=1$, $2, \cdots$, the zeros of $F_{n}(z)$ lie in $H(K)$, the convex hull of $K$. I will show in this paper that $H(K)$ contains all the zeros of $F_{n}^{\prime}(z), n=1,2, \cdots$.

2. Let

$$
z=g(w)=w+b_{0}+b_{-1} w^{-1}+\cdots
$$

be the inverse function of $w=f(z)$. This is a schlicht function mapping the exterior of $\Sigma_{w}$ onto $\tilde{K}$. Since $g(w)$ is bounded in $\{w: 1<|w|<R\}, R>1$, $\lim _{r \rightarrow 1^{+}} g\left(r e^{i \theta}\right)$ exists for almost all $\theta, 0 \leqq \theta \leqq 2 \pi$. Let $g(\theta)$ be a function defined as this limit, where it exists, and as a value lying in $K$ for other values of $\theta$. Then, because of the schlichtness of $g(w)$, all the values of $g(\theta)$ lie in $K$. Finally, using Lebesgue's theorem of bounded convergence, we find that

$$
\begin{aligned}
\frac{1}{2 \pi} \int_{0}^{2 \pi} g(\theta) e^{-i n \theta} d \theta & =b_{n}, & & n=0,-1, \cdots, \\
& =1, & & n=1, \\
& =0, & & n=2,3, \cdots .
\end{aligned}
$$

Received by the editors October 22, 1971.

AMS 1970 subject classifications. Primary 33A08.

Key words and phrases. Faber polynomials, zeros of Faber polynomials, zeros of the derivatives of Faber polynomials. 
3. Faber [1] showed that

$$
\frac{g^{\prime}(w)}{g(w)-z}=\sum_{n=0}^{\infty} \frac{F_{n}(z)}{w^{n+1}}
$$

and from this we easily obtain

$$
\frac{1}{g(w)-z}=\sum_{n=1}^{\infty} \frac{F_{n}^{\prime}(z)}{n w^{n}} .
$$

4. Our object now is to get another representation for $F_{n}^{\prime}(z)$ by regarding $(g(w)-z)^{-1}$ as the reciprocal of a power series, namely

$$
\frac{1}{g(w)-z}=\frac{1}{w+\left(b_{0}-z\right)+b_{-1} w^{-1}+\cdots} .
$$

For a power series $\sum_{n=0}^{\infty} c_{n} z^{n}$, it is readily found that if $\sum_{n=0}^{\infty} d_{n} z^{n}=$ $\left(\sum_{n=0}^{\infty} c_{n} z^{n}\right)^{-1}$, then

$$
d_{n}=(-1)^{n} \frac{\left|\begin{array}{llll}
c_{1} & c_{0} & 0 & \\
c_{2} & c_{1} & c_{0} & 0 \\
& & & c_{0} \\
c_{n} & & c_{1}
\end{array}\right|}{c_{0}^{n+1}}
$$

We can apply (6) to (5) and use (4) to obtain

$$
\frac{F_{n+1}^{\prime}(z)}{n+1}=(-1)^{n}\left|\begin{array}{ccccr}
b_{0}-z & 1 & 0 & & \cdot \\
b_{-1} & b_{0}-z & 1 & 0 & \cdot \\
& & & & 1 \\
b_{-(n-1)} & & & b_{0}-z
\end{array}\right|
$$

Thus we see that the zeros of the derivatives of Faber polynomials are of the eigenvalues a type of matrix known as a Toeplitz matrix.

5. If $\left(e_{p, q}\right)$ is an $n$th order matrix and if $\lambda$ is an eigenvalue, then there is a nondegenerate vector $\left(x_{1}, \cdots, x_{n}\right)$ such that

$$
\sum_{q=1}^{n} e_{p, q} x_{q}=\lambda x_{p}, \quad p=1, \cdots, n .
$$


From this we obtain

$$
\sum_{p=1}^{n} \sum_{q=1}^{n} e_{p, q} x_{q} \bar{x}_{p}=\lambda \sum_{p=1}^{n}\left|x_{p}\right|^{2} .
$$

Now, in the case of (7), we have $e_{p, q}=b_{q-p}$, if we understand that $b_{1}=1$ and $b_{n}=0$ for $n=2,3, \cdots$. Thus if $z_{0}$ is a zero of $F_{n+1}^{\prime}(z)$ we find that there is a nondegenerate vector $\left(x_{1}, \cdots, x_{n}\right)$ such that

$$
\sum_{p=1}^{n} \sum_{q=1}^{n} b_{q-p} x_{q} \bar{x}_{p}=z_{0} \sum_{p=1}^{n}\left|x_{p}\right|^{2} .
$$

Using (2) this becomes

$$
\begin{aligned}
\frac{1}{2 \pi} \int_{0}^{2 \pi} g(\theta) \mid x_{1}+x_{2} e^{-i \theta} & +\cdots+\left.x_{n} e^{-i(n-1) \theta}\right|^{2} d \theta \\
& =z_{0} \frac{1}{2 \pi} \int_{0}^{2 \pi}\left|x_{1}+x_{2} e^{-i \theta}+\cdots+x_{n} e^{-i(n-1) \theta}\right|^{2} d \theta .
\end{aligned}
$$

This shows that $z_{0}$ is a weighted arithmetic average of the values in the essential range of $g(\theta)$, and thus lies in the convex hull of the essential range of $g(\theta)$. We have already noted that the values of $g(\theta)$ lie in $K$, and thus the convex hull of the essential range of $g(\theta)$ lies in the convex hull of $K$, and our proof that $z_{0}$, a zero of $F_{n+1}^{\prime}(z)$, lies in the convex hull of $K$ is complete. The results of this paper now permit us to apply the results of [2] to the zeros of the derivatives of Faber polynomials. In addition, the methods of [2] can be applied to obtain results on the asymptotic distribution of the Faber polynomials themselves.

\section{REFERENCES}

1. G. Faber, Über polynomische Entwickelungen, Math. Ann. 57 (1903), 389-408.

2. J. L. Ullman, Toeplitz matrices associated with a semi-infinite Laurent series, Proc. London Math. Soc. 22 (1971), 164-192.

Department of Mathematics, University of Michigan, Ann Arbor, Michigan 48104 